\title{
Independent Predictors of Early Death of Polytrauma Patients: An Analysis of 696 Patients
}

\author{
Ladislav Mica $^{1 *}$, Katja Albrecht ${ }^{1}$, Marius Keel2 and Otmar Trentz ${ }^{3}$
}

${ }^{1}$ Division of Trauma Surgery, University Hospital of Zürich, Switzerland

${ }^{2}$ University Hospital of Othopedic Surgery, Inselspital Bern, Switzerland

${ }^{3}$ Former Head of the Department of Trauma Surgery, University Hospital of Zürich, Switzerland

\begin{abstract}
Polytrauma patients are at high risk to die due to acute physiological deterioration after a high injury load within the first hours. Trauma scores such as ISS, NISS and APACHE II and laboratory parameters were collected at admission. The aim of this study was to evaluate the independent predictors of early death of the polytrauma patient.

A total of 696 patients (age $40.8 \pm 15.5$ years) who had an ISS $\geq 17$ and were admitted to a trauma center within the first 24 hours after trauma were included into this retrospective study. The patients were subdivided into survivors and those who died within the first 72 hours. ISS, NISS and APACHE II score, and laboratory parameters such as lactate, prothrombin time, $\mathrm{pH}$-value, hematocrite, middle arterial pressure and platelets were collected at admission time. Data were compared with Mann-Whitney test and $x^{2}$-test for proportions. The data were considered as significant if $p<0.05$. Predictive ability was evaluated by using receiver operating characteristic curves. Independent predictors were analyzed by logistic regression analysis.
\end{abstract}

Significantly increased values of ISS, NISS, APACHE II and serum lactate, and significantly decreased values of prothrombin time, middle arterial pressure, hematocrite, Platelets and $\mathrm{pH}$-value were found in the non-survivor group $(p<0.001)$. APACHE II, NISS, ISS score and the prothrombin time were found to be independent predictive values of death.

Early recognition of the risk of death followed by multidisciplinary resuscitation efforts could lead to a better survival rate in the population of polytrauma patients.

Keywords: Polytrauma; ISS; NISS; APACHE II; Prothrombin time

\section{Introduction}

The availability of circulatory supportive medication and scheduled surgical strategies for the resuscitation of polytrauma patients led to a better survival outcome. Trauma scoring systems developed during the last decades define the threshold of a polytrauma but do not serve as predictive values of death. Independent predictors of an early death among the widely used trauma scoring systems and commonly available laboratory parameters such as lactate, $\mathrm{pH}$, prothrombin time, platelets and hematocrite are not defined yet. The question still remains, which of the commonly used parameters are the most independent predictors of patients' early death after suffering a polytrauma. The aim is to improve resuscitative procedures and to prevent patient's early death as a multidisciplinary task.

Trauma scoring systems such as ISS, NISS, and APACHE II define the level of a polytrauma but do not serve as predictive values of early death. ISS and NISS describe the severity of trauma depending on the anatomical region and injury pattern. The injury pattern describes indirectly the severity of trauma, rendering the patient to higher risk of developing trauma associated diseases such as SIRS. APACHE II score uses different physiological and laborytory parameters at admission to describe the severity of the trauma load on the organism. However, all polytrauma patients are at high risk to develop an acute coagulopathy of trauma shock $[1,2]$ in the first few hours after suffering the trauma. There [3] was a likelihood of $98 \%$ found to develop a coagulopathy if the ISS was above 25 points, the $\mathrm{pH}$-value below 7.1, the core temperature under $34^{\circ} \mathrm{C}$ and the systolic blood pressure under $70 \mathrm{mmHg}$ [3]. The traditional description depicts the acute coagulopathy of trauma shock [1] as a later event mainly caused by resuscitative attempts occurring in the hospital. There are patients admitted to the emergency room with an already established or evolving coagulopathy due to trauma. Similar cases were reported in a retrospective study of 1088 [4] patients and confirmed by other retrospective studies with high case loads $[5,6]$. In these studies was a strong correlation between coagulopathy and mortality found, and coagulopathy was identified as an independent risk factor [4]. Acute coagulopathy of trauma shock was found to be associated with longer ICU stays [7], with higher risk to develop acute renal failure and multiple organ failure (MOF) [7] and with a trend towards acute respiratory distress syndrome [7]. In all these studies the transportation time was short and resuscitative fluid management was not excessive. Only few studies show the threshold values when to use blood products for resuscitation in the polytrauma patients and when to use normal saline [8] depending on ISS, NISS or APACHE II score. Independent predictors of the early death are not clearly defined, yet. Little is found in the literature showing clearly a benefit for survival after early and aggressive resuscitation with blood products in dependence of ISS, NISS or APACHE II score on one hand. On the other hand there seems to be a significant risk to develop an iatrogenic SIRS using blood-products for resuscitation as shown earlier [9]. This dilemma is

*Corresponding author: Ladislav Mica, Department of Trauma Surgery, University Hospital of Zürich, 8091 Zürich, Switzerland, Tel: 41/ 442554198 E-mail: ladislav.mica@usz.ch

Received February 06, 2012; Accepted March 09, 2012; Published March 05, 2012

Citation: Mica L, Albrecht K, Keel M, Trentz O (2012) Independent Predictors of Early Death of Polytrauma Patients: An Analysis of 696 Patients. J Trauma Treatment 1:118. doi:10.4172/2167-1222.1000118

Copyright: (c) 2012 Mica L, et al. This is an open-access article distributed unde the terms of the Creative Commons Attribution License, which permits unrestricted use, distribution, and reproduction in any medium, provided the original author and source are credited. 
not solved, yet, but the aim is clear: avoid death of the patient. An early identification of critically incipient trauma coagulopathy according to trauma scoring systems and laboratory parameters could lead to a lesser mortality and morbidity in the polytrauma patient.

In this study the focus was set on widely used trauma scoringsystems as a source for the severity of trauma and laboratory parameters as a sign of an early physiological deterioration. Commonly used laboratory parameters were analyzed which may guide physicians' decisions during the initial management of a polytrauma patient. The differences in clotting components, i.e. platelets, prothrombin time, the values of serum lactate and $\mathrm{pH}$ and whole blood parameters such as hematocrite and middle arterial pressure (MAP) were analyzed and compared with the values of survivors and non survivors over the first 72 hours. To provide useful information about the predictive value of widely used scoring systems, APACHE II, ISS and NISS scores were analysed. The aim was to determine independent predictive values of death within the first 72 hours of a polytrauma patient at admission.

\section{Materials and Methods}

\section{Patients}

696 polytrauma patients admitted to the emergency room of the University Hospital of Zürich in the time period of the year 19962002 were enclosed into this study. Admission criteria were an injury severity score (ISS) $\geq 17$ points, age $\geq 16$ years and an admission time at least $24 \mathrm{~h}$ after suffering the polytrauma. The patients were subjected to intensive care treatment and damage control surgery. The population was subdivided into two groups: survivors and non survivors of the first 72 hours. All patients' data were collected retrospectively. ISS, NISS and APACHE II were defined according to the data collected at admission to the emergency department. All data were retrieved from patient's sheet according the local Institutional Review Board (IRB) approval according to the University of Zürich IRB guidelines and was conducted according to the guidelines of good clinical practice (Retrospektive Analysen in der Chirurgischen Intensivmedizin Nr. StV 01-2008).

\section{Surgical treatment}

The treatment of all admitted patients followed the ATLS guidelines and the previously assessed trauma-management protocol [10-12]. Briefly, after the airway, ventilation and cardiovascular management life saving surgery with decompression of body-cavities control of hemorrhage and contaminated tissue took place. The first surgical interventions were followed by stabilization of major fractures and radical debridement of death tissue. Cefalozoline was used as peri-operative antibiotics. In all of the admitted patients an enteral nutrition was established within $24 \mathrm{~h}$ after trauma to avoid spontaneous transmigration of the enteral microbial flora and peritoneal contamination.

\section{Trauma scoring systems}

The Injury Severity Score (ISS) [13] and the New Injury Severity Score (NISS) [14] were used to define the severity of trauma. The Acute Physiology and Chronic Health Evaluation, APACHE II [15] score, was used to evaluate the overall physiological impairment of the patient.

\section{Laboratory parameters}

Lactate, $\mathrm{pH}$ and hematocrite were measured in intervals with a blood-gas analyzer (ABL 800 Flex, Radiometer GmbH, Thalwil, $\mathrm{CH}$ ). Platelets were measured by flow cytometry (Facscalibur, Becton
Dickinson, Allschwil, $\mathrm{CH}$ ). The prothrombin time was measured by a standardized previously described method [16].

\section{Statistical analysis}

Continuous data are presented as mean \pm standard deviation (SD) and are compared between groups using the Mann-Whitney test. For proportions the $\chi^{2}$-test was used. All statistical computations were performed using SPSS 17.0 (SPSS Inc., Chicago, IL, USA). Results were considered significant at $\mathrm{p}<0.05$. Predictive ability of variables was reported as area under the ROC-curve (AUC) \pm standard error. Independent predictors were analyzed by stepwise (forward and backward) logistic regression analysis.

\section{Results}

\section{Patient population}

Totally 696 patients with an average age of $40.8 \pm 15.5$ years were included into this study. 528 were males and 168 were females. The overall admission time was $2.0 \pm 1.9$ hours $(2.1 \pm 2.2$ vs. $1.6 \pm 0.9$, survivor vs. non-survivor, $\mathrm{p}=0.028)$. Totally $184(26.4 \%$ of all $)$ patients died within the first 72 hours, 135 (27.6\% of males) males and 48 (28.4\% of females) females (Table 1 ).

\section{ISS, NISS and APACHE II}

The overall ISS of the admitted patients was $35 \pm 12.6$ points and the overall NISS was $43.6 \pm 12.3$ points (Table 1$)$. The ISS $(32.4 \pm 11.8$ vs. $37.8 \pm 13.4$, survivor vs. non-survivor, $\mathrm{p}<0.001)$ and NISS $(41.4 \pm$ 13.5 vs. $50.2 \pm 14.1$, survivor vs. non-survivor, $\mathrm{p}<0.001)$ scores were significantly elevated in the patients who did not survive the first 72 hours (Table 1). Similar predictive powers of ISS (AUC $0.61 \pm 0.03$; Odds-Ratio 0.96) and NISS (AUC $0.68 \pm 0.03$; Odds-Ratio 1.04) were found (Figure 1). APACHE II (15.2 \pm 7.9 vs. $25.3 \pm 7.7$, survivor vs. non-survivor, $\mathrm{p}<0.001)$ score evaluated at admission was significantly elevated in patients who died within the first 72 hours (Table 1). A high predictive power (AUC $0.80 \pm 0.02$; Odds-Ratio 1.15) of the APACHE II score was found at admission (Figure 1).

\section{Lactate acidosis at admission}

Serum lactate $(3.0 \pm 2.3$ vs. $5.6 \pm 3.9$, survivor vs. non-survivor, $\mathrm{p}<0.001)$ was evaluated at admission for each patient. The lactate values (AUC $0.73 \pm 0.03$ ) (Figure 2 ) were significantly elevated in patients who did not survive the first 72 hours (Table 1). The serum

\begin{tabular}{|l|l|l|l|}
\hline Characteristics & $\begin{array}{l}\text { Survival over 3 } \\
\text { days }\end{array}$ & Death within 3 days & p-value \\
\hline Number [\% of total] & $512(73.6 \%)$ & $184(26.4 \%)$ & ns. $^{\dagger}$ \\
Age [years] & $39.1 \pm 16.6$ & $45.5 \pm 19.2$ & $<_{0.001^{*}}$ \\
Males [\% of Males] & $393(74.4 \%)$ & $135(27.6 \%)$ & ns. $^{\dagger}$ \\
Females [\% of Females] & $121(71.6 \%)$ & $48(28.4 \%)$ & ns. $^{\dagger}$ \\
ISS [points] & $32.4 \pm 11.8$ & $37.8 \pm 13.4$ & $<0.001^{*}$ \\
NISS [points] & $41.4 \pm 13.5$ & $50.2 \pm 14.1$ & $<0.001^{*}$ \\
APACHE II[points] & $15.2 \pm 7.9$ & $25.3 \pm 7.7$ & $<0.001^{*}$ \\
Lactate [mmol/L] & $3.0 \pm 2.31$ & $5.6 \pm 3.9$ & $<0.001^{*}$ \\
pH & $7.31 \pm 0.13$ & $7.2 \pm 0.2$ & $<0.001^{*}$ \\
Platelets & $192 \pm 72.5$ & $152 \pm 79.6$ & $<0.001^{*}$ \\
Prothrombin Time [\%] & $80.5 \pm 19.3$ & $58.9 \pm 23.6$ & $<0.001^{*}$ \\
Haematocrite & $33.4 \pm 7.8$ & $26.4 \pm 79.6$ & $<0.001^{*}$ \\
MAP & $93.2 \pm 37.1$ & $79.7 \pm 30.0$ & $<0.001^{*}$ \\
Admission time [h] & $2.1 \pm 2.2$ & $1.6 \pm 0.9$ & $0.028^{*}$ \\
\hline
\end{tabular}

*Mann-Whitney Rank Sum test, ${ }^{\dagger} X^{2}$-test.

Table 1: Characteristics of patient population (ISS: Injury Severity Score, NISS New Injury Severity Score, APACHE II: Acute Physiology and Chronic Health Evaluation). Data are given as mean $\pm S D$, significance was set at $p<0.05$, the data in parentheses are percentage values. ${ }^{*}$ Mann-Whitney test, ${ }^{\dagger} \mathrm{X}^{2}$-test. 


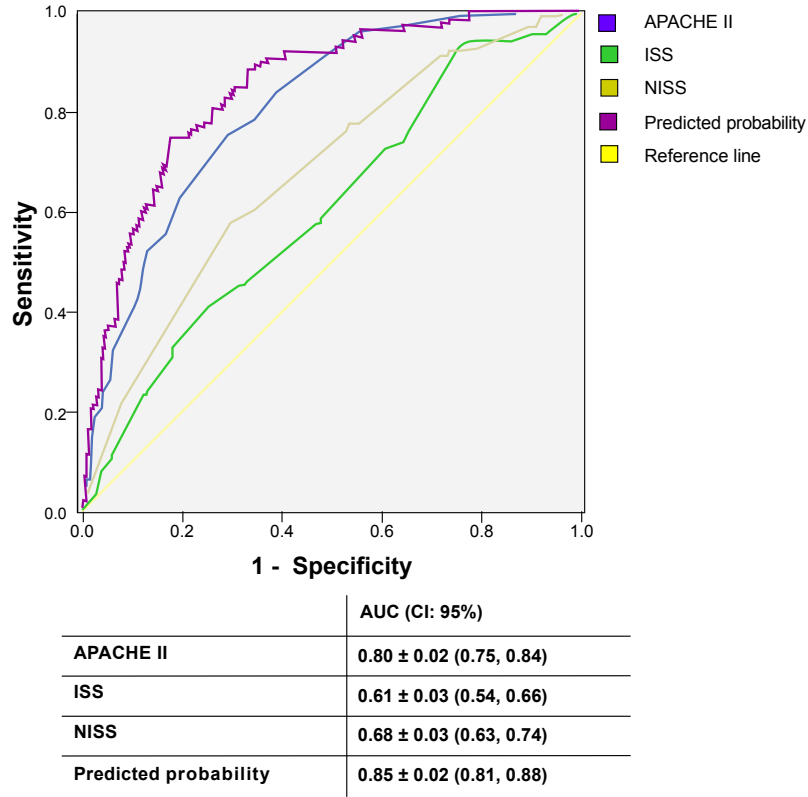

Figure 1: ROC curve of APACHE II (AUC $0.80 \pm 0.02$; Odds-Ratio 1.15), ISS (AUC $0.61 \pm 0.03$; Odds-Ratio 0.96), and NISS (AUC $0.68 \pm 0.03$; Odds-Ratio 1.04). The patients were evaluated by the scoring systems ad admission. All scores of the patients who did not survive were significantly elevated $(p<$ $0.001)$. Predicted probability of death based on all four independent predictors (APACHE II, ISS, NISS and prothrombin time (Figure 3.)) results in a very good predictive probability. Data are given as area under the curve (AUC) and 95\% confidence interval (Cl: 95\%) with it's with (lower bound, upper bound).

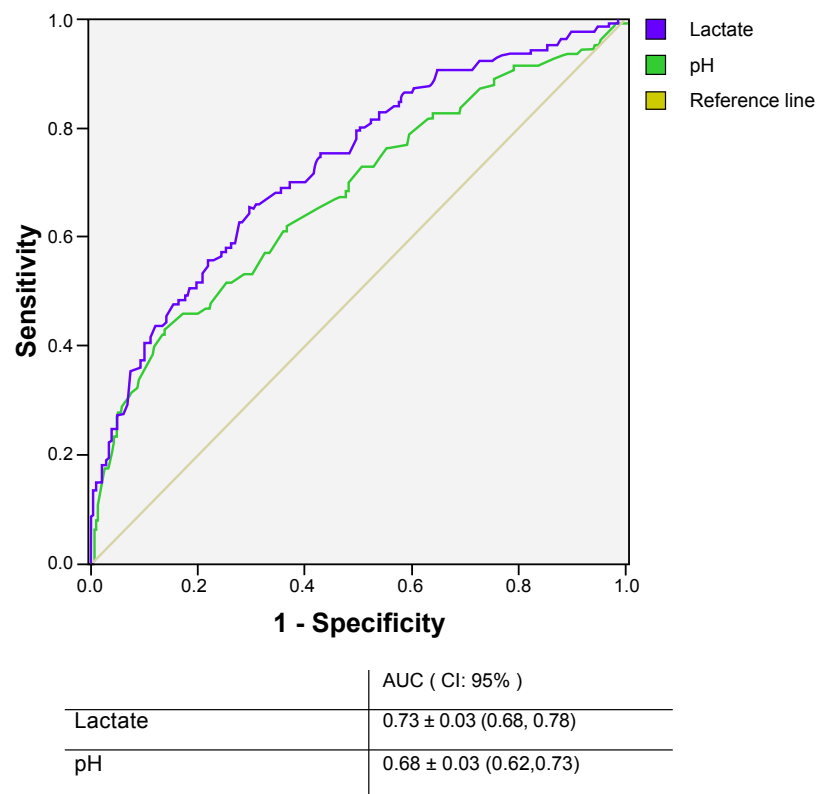

Figure 2: ROC curve of serum lactate and $\mathrm{pH}$ values, measured ad admission Both values were significantly different between surviving and non-surviving patients $(\mathrm{p}<0.001)$. Thus, the predictive ability of serum lactate and $\mathrm{pH}$-value revealed no independency in the multiple logistic regression analysis. The predictive ability of $\mathrm{pH}$ and lactate is quite poor; hence $\mathrm{pH}$ is already contained in the APACHE II score. Data are given as area under the curve (AUC) and 95\% confidence interval ( $\mathrm{Cl}$ : 95\%) with it's with (lower bound, upper bound).

lactate concentrations were normalized after resuscitation and damage control surgery after 24 hours. There were no significant differences of lactate values found between groups after 24 hours. The $\mathrm{pH}$ values ( 7.31 \pm 0.1 vs. $7.2 \pm 0.2$, survivor vs. non-survivor, $\mathrm{p}<0.001$ ) (AUC $0.68 \pm$ 0.03 ) (Figure 2) measured at admission were significantly reduced in patients who did not survive (Table 1).

\section{Platelets and prothrombin time}

Significantly decreased platelets (192 \pm 75.5 vs. $152 \pm 79.6$, survivor vs. non-survivor, $\mathrm{p}<0.001$ ) (AUC $0.62 \pm 0.03$ ) and prothrombin times ( $80.5 \pm 19.3$ vs. $58.9 \pm 23.6$, survivor vs. non-survivor, $\mathrm{p}<0.001$ ) (AUC $0.76 \pm 0.02$; Odds-Ratio 0.98 ) (Figure 3 ) were found in patients who did not survive the first 72 hours (Table 1). After resuscitation and damage control surgery the values were mostly normalized without significant differences after 24 hours (data not shown).

\section{Blood loss}

Haematocrite-values $(33.4 \pm 7.8$ vs. $26.4 \pm 7.9$, survivor vs. nonsurvivor, $\mathrm{p}<0.001)$ and middle arterial blood pressure (MAP) $(93.2$ \pm 37.1 vs. $79.7 \pm 30.0$, survivor vs. non-survivor, $\mathrm{p}<0.001$ ) were measured at admission of the patients. As expected, these values were significantly (Table 1 ) reduced in patients who died within the first 72 hours (Haematocrite: AUC $0.64 \pm 0.03$, MAP: AUC $0.65 \pm 0.03$ ) (Figure 4).

\section{Independent predictors of death: ISS, NISS, APACHE II and prothrombin time}

In a stepwise logistic regression including all significant parameters above, APACHE II, NISS, ISS score and the prothrombin time were found to be independent predictors of death. The combination "predicted probability" of these variables thus is a significantly better predictor than each single score (AUC $0.85 \pm 0.02$ ) (Figure 1)

\section{Discussion}

Massive bleeding is the key issue in trauma and emergency surgery

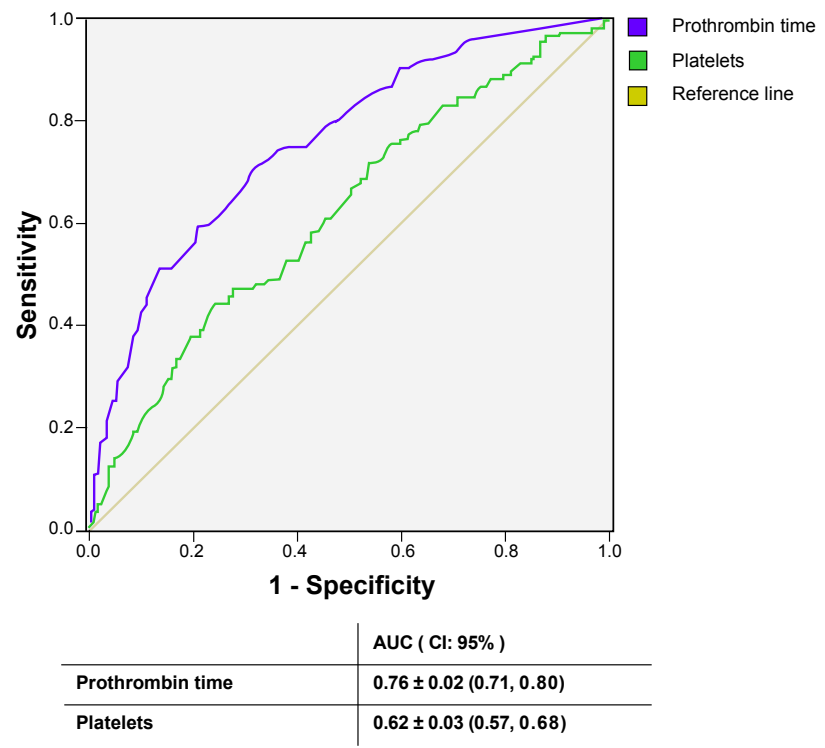

Figure 3: ROC curve of prothrombin time and platelets, measured ad admission. Both parameters were significantly different between survivors and non-survivors $(p<0.001$ ). Prothrombin time (AUC $0.76 \pm 0.02$; Odds-Ratio 0.98 ) evolved as an independent predictive value of death within the first 72 hours. In the case of a polytrauma patient the prothrombin time has a higher predictive value than platelet count. Data are given as area under the curve (AUC) and 95\% confidence interval (Cl: 95\%) with it's with (lower bound, upper bound). 


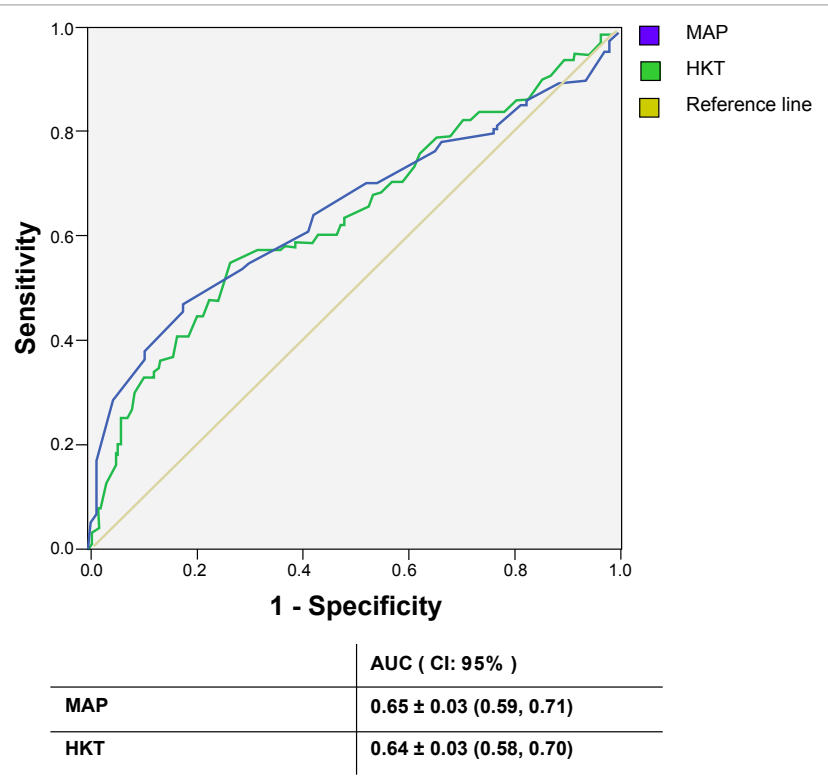

Figure 4: ROC curve of middle arterial pressure (MAP) and hematocrite (HKT), measured at admission. Both parameters were significantly different between survivors and non-survivors $(p<0.001)$. The data revealed poor predictive value of MAP and HKT, certainly when massively lowered death might be unenviable. Data are given as area under the curve (AUC) and $95 \%$ confidence interval (Cl: 95\%) with it's with (lower bound, upper bound).

$[17,18]$. The availability of resuscitative components such as red blood cells, platelets and fresh frozen plasma does not guarantee the survival of a polytrauma patient with a massive hemorrhage after suffering a trauma. The data presented in this study show independent predictive parameters such as ISS, NISS, APACHE II and prothrombin time for the posttraumatic death within the first 72 hours after admission to a level I trauma center. Probably, pointing on decision guiding values. Normally, the operative mortality is low for most of the surgical interventions, ranging from $<0.1 \%$ to $8 \%$ [19]. Unexpected acute bleeding during surgical interventions may raise the mortality over $20 \%$ [19]. Literature lacks clear evidenced procedures and independent predictive parameters to estimate the risk of early death and to avoid hemorrhage in polytrauma patients. This seems not to be very surprising due to the wide heterogeneity of this field.

\section{ISS and NISS: tissue destruction bleeds}

Trauma leads to destruction of tissue and blood loss. The demonstrated data show significantly higher values in both scoring systems comparing the survival to the non-survival group, according to the severity of tissue destruction. Tissue destruction leads to the set free of the Kininogene and Kallikrein leading to the activation of the factor XII (Hageman-factor) [20]. Systemic spread of Kininogene and Kallikrein may activate systemically the factor XII leading to a consumptive coagulopathy. However, the mechanism how trauma coagulopathy develops remains uncertain. Other theories point on the rise of soluble thrombomodulin and fall in protein $\mathrm{C}$ levels after trauma [20]. This may be correlated with the development of acute coagulopathy of trauma shock [1]. Protein C is a natural anticoagulant excessively consuming plasminogen activator inhibitor (PAI-1) [20,2]. The correlation between rise of PAI-1 and decrease of protein C level were shown elsewhere [2]. Theoretically the increase in membranebound thrombomodulin on endothelial cells may lead to scavenging of thrombin, reducing its amount to produce fibrin and raising the amount of activated protein $\mathrm{C}$ [21] and thus inhibiting the clotting cascade. However, these assumptions still remain speculative.

\section{APACHE II: physiological changes due to traumatic hemorrhage and tissue destruction}

Metabolic lactate acidosis accompanied by hypothermia lowers the functionality of blood clotting factors supporting progressive bleeding. The activity of the prothrombinase complex (FXa/FVa) is reduced by $50 \%$ at $\mathrm{pH} 7.2$, by $70 \%$ at $\mathrm{pH} 7.0$ and by $90 \%$ at $\mathrm{pH} 6.8$ $[22,23]$. Most of the factors involved in blood clotting are severely impaired in function when the $\mathrm{pH}$ is lower than 7.1: probably due to reduced affinity of $\mathrm{Ca}^{2+}$ binding sites. Hypothermia inhibits any enzymatic activity according to the equation of Arrhenius and van't Hoff and dilution during resuscitation and hypothermia favors ongoing bleeding. Hypothermia supports lactate acidosis by the above mentioned mechanism. Clinically significant changes in blood clotting are already observed at temperatures under $34^{\circ} \mathrm{C}\left(93.2^{\circ} \mathrm{F}\right)[23,24]$ even if the concentration of clotting factors has no pathological changes. When the core temperature falls below $33^{\circ} \mathrm{C}\left(91.4^{\circ} \mathrm{F}\right)$ in a polytrauma patient the activity of the blood-clotting system falls below $50 \%$ even if there is a normal factor concentration and functionality [24]. Higher APACHE II scores significantly increased the risk of death within the first 72 hours after admission. Early and aggressive resuscitative efforts with restoring patient's physiology especially blood coagulation and acidosis could improve the survival outcome.

\section{Acidosis}

Lactate acidosis in the polytrauma patient roots in several problems: hypothermia, anemia and lactate acidosis itself. The kidney and the respiratory system usually fail to correct the acidosis in polytrauma and shocked patients. Corrective resuscitative interventions with e.g. sodium bicarbonate $\left(\mathrm{NaHCO}_{3}\right)$ and tris-hydroxymethylaminomethane (THAM) may restore normal $\mathrm{pH}$, furthermore, they seems to have a thrombo-permissive effect $[25,26]$. Acidosis increases the fibrinogen degradation by the factor 1.8 and thus leads to prolonged bleeding [27]. Parallely, the platelets are also inhibited by lover blood $\mathrm{pH}$. However, how acidosis leads to decreased thrombocyte counts is not fully understood, yet. Transfusion of packed red blood cells may aggravate posttraumatic acidosis due to a lower base excess acquired during storage time [28]. There seems to be a general inhibition of all cellular functions during acidosis. Even when the $\mathrm{pH}$-values reached a similar level after 24 hours the suppressive effect on physiological functions of acidosis could be long-termed.

\section{Platelets}

There is evidence to keep platelet counts above $50-100 \times 10^{9} / \mathrm{L}$ in the polytrauma patient [29]. The shown platelet counts in this study show levels above $100 \times 10^{9} / \mathrm{L}$ also in patients who died within the first 72 hours after admission. Platelets and red blood cells have to be in a certain relation to allow optimal thrombocyte function. Thrombocyte function is reduced during acidosis with a permissive effect on ongoing bleeding. Thombocytes measured after 24 hours (data not shown) had similar values in all groups pointing on a possible long-term effect, probably caused by acidosis. Platelet loss may be attributed to consumption at trauma site and to loss of whole blood.

\section{Prothrombin time}

Significant reduction of the prothrombin time in patients who did not survive may be caused by hypothermia, acidosis and dilution or activation-decay [30]. The main reason for the reduction of the values 
cannot be identified here. The acidosis correlates with the reduction of the prothrombin time, as discussed earlier. Dilution of the blood coagulation cascade during resuscitative attempts has not to be considered due to very low rescue-times and low resuscitative volumes of cristaloids. Further more, the loss of whole blood and acidosis seem to be the core reasons for reduced prothrombin times and for the independence as a predictor of death.

\section{Hematocrite}

The decreased hematocrite values favor the development of acidosis. Lowered haematocrite is a double cutting edge, it favors hypoxemia and an anaerobic metabolism resulting in lactate acidosis and the reduction of haemoglobin reduces also the main blood buffer system [31]. The data shown in this study suggest hemorrhage as a death factor. Taken to gather lower hematocrite-values favor the genesis of lactate acidosis [31], acidosis itself inhibits the blood coagulation cascade and probably also the normal function of platelets. There is evidence that early and aggressive resuscitation with FFP (fresh frozen plasma) and red blood cells (RBC) improves the ICU outcome of the polytrauma patient $[32,33]$. The very early normalization of the bloodclotting pathway could lead to a better survival ratio as seen in military trauma settings [33].

\section{Conclusions}

The APACHE II, ISS and NISS scores with the prothrombin time as independent predictive values of death can be used to guide surgeons' decisions during the management of an acute polytrauma patient. This data show that the very early management of polytrauma patient, according to the independent predictive factors could improve the outcome of the polytrauma patient. This proves the 'golden hour' in the treatment of trauma shock.

\section{References}

1. Frith D, Brohi $K(2010)$ The acute coagulopathy of trauma shock: clinical relevance. Surgeon 8: 159-163.

2. Brohi K, Cohen MJ, Ganter MT, Matthay MA, Mackersie RC, et al. (2007) Acute traumatic coagulopathy: initiated by hypoperfusion: modulated through the protein C pathway? Ann Surg 245: 812-818.

3. Cosgriff N, Moore EE, Sauaia A, Kenny-Moynihan M, Burch JM, et al. (1997) Predicting life-threatening coagulopathy in the massively transfused trauma patient: hypothermia and acidoses revisited. J Trauma 42: 857-861.

4. Brohi K, Singh J, Heron M, Coats T (2003) Acute traumatic coagulopathy. J Trauma 54: 1127-1130

5. MacLeod JB, Lynn M, McKenney MG, Cohn SM, Murtha M (2003) Early coagulopathy predicts mortality in trauma. J Trauma 55: 39-44.

6. Maegele M, Lefering R, Yucel N, Tjardes T, Rixen D, et al. (2007) Early coagulopathy in multiple injury: an analysis from the German trauma registry on 8724 patients. Injury 38: 298-304.

7. Salim A, Martin M, Constantinou C, Sangthong B, Brown C, et al. (2006) Acute respiratory distress syndrome in the trauma intensive care unit: Morbid but not mortal. Arch Surg 141: 655-658.

8. Hagiwara A, Kimura A, Kato H, Mizushima Y, Matsuoka T, et al. (2010) Hemodynamic reactions in patients with hemorrhagic shock from blunt trauma after initial fluid therapy. J Trauma 69: 1161-1168.

9. Beale E, Zhu J, Chan L, Shulman I, Harwood R, et al. (2006) Blood transfusion in critically injured patients: a prospective study. Injury $37: 455-465$.

10. Ertel W, Trentz O (1995) Causes of shock in the severely traumatized patient: emergency treatment. In: Goris RJA, Trentz O The integrated approach to trauma care, the first 24 hours. Springer 78-87.

11. Trentz O, Friedl HP (1995) Therapeutic sequences in the acute period in unstable patients. In: Goris RJA, Trentz O The integrated approach to trauma care, the first 24 hours. Springer 172-178.

12. Collicott PE, Hughes I (1980) Training in advanced trauma life support. JAMA 243: $1156-1159$

13. Baker SP, O'Neill B, Haddon W Jr, Long WB (1974) The injury severity score: A method for describing patients with multiple injuries and evaluating emergency care. J Trauma 14: 187-196.

14. Champion HR, Copes WS, Sacco WJ, Lawnick MM, Bain LW, et al. (1990) A new characterization of injury severity. J Trauma 30: 539-545.

15. Knaus WA, Draper EA, Wagner DP, Zimmerman JE (1985) APACHE II: a severity of disease classification system. Crit Care Med 13: 818-829.

16. Jackson CM, White GC (2001) Scientific and standardization committee communication: a reference system approach to future standardization of laboratory tests for hemostasis.

17. Sauaia A, Moore FA, Moore EE, Lezotte DC (1996) Early risk factors for postinjury multiple organ failure. World J Surg 20: 392-400.

18. Stewart RM, Myers JG, Dent DL, Ermis P, Gray GA, et al. (2003) Seven hundred fifty-three consecutive deaths in a level 1 trauma center: the argument for injury prevention. J Trauma 54: 66-70.

19. Copeland GP, Jones D, Walters M (1991) POSSUM: a scoring system for surgical audit. Br J Surg 78: 355-360.

20. Bryant JW, Shariat-Madar Z (2009) Human plasma kallikrein-kinin system physiological and biochemical parameters. Cardiovasc Hematol Agents Med Chem 7: 234-250.

21. Roberts HR, Hoffman M, Monroe DM (2006) A cell-based model of thrombin generation. Semin Thromb Hemost 32: 32-38.

22. Meng ZH, Wolberg AS, Monroe DM 3rd, Hoffman M (2003) The effect of temperature and $\mathrm{pH}$ on the activity of factor VIla: implications for the efficacy of high-dose factor VIla in hypothermic and acidotic patients. J Trauma 55: 886-891.

23. Lier H, Krep H, Schroeder S, Stuber F (2008) Preconditions of hemostasis in trauma: a review. The influence of acidosis, hypocalcemia, anemia, and hypothermia on functional hemostasis in trauma. J Trauma 65: 951-960.

24. Hess JR, Brohi K, Dutton RP, Hauser CJ, Holcomb JB, et al. (2008) The coagulopathy of trauma: a review of mechanisms. J Trauma 65: 748-754.

25. Martini WZ, Dubick MA, Wade CE, Holcomb JB (2007) Evaluation of tris hydroxymethylaminomethane on reversing coagulation abnormalities caused by acidosis in pigs. Crit Care Med 35: 1568-1574.

26. Martini WZ, Dubick MA, Pusateri AE, Park MS, Ryan KL, et al. (2006) Does bicarbonate correct coagulation function impaired by acidosis in swine? Trauma 61: 99-106.

27. Martini WZ (2009) Coagulopathy by hypothermia and acidosis: mechanisms of thrombin generation and fibrinogen availability. J Trauma 67: 202-208.

28. Raat NJ, Berends F, Verhoeven AJ, de Korte D, Ince C (2005) The age of stored red blood cell concentrates at the time of transfusion. Transfus Med 15 : 419-423.

29. Rossaint R, Bouillon B, Cerny V, Coats TJ, Duranteau J, Ferna, et al. (2010) Management of bleeding following major trauma: an updated European guideline. Crit Care 14: R52.

30. Bolliger D, Szlam F, Levy JH, Molinaro RJ, Tanaka KA (2010) Haemodilutioninduced profibrinolytic state is mitigated by fresh-frozen plasma: implications for early haemostatic intervention in massive haemorrhage. Br J Anaesth 104 318-325.

31. Chiarla C, Giovannini I, Giuliante F, Vellone M, Ardito F, et al. (2010) Significance of hemoglobin concentration in determining blood $\mathrm{CO}_{2}$ binding capacity in critical illness. Respir Physiol Neurobiol 172: 32-36.

32. Sihler KC, Napolitano LM (2009) Massive transfusion: new insights. Chest 136 1654-1667.

33. Kashuk JL, Moore EE, Johnson JL, Haenel J, Wilson M, et al. (2008) Postinjury life threatening coagulopathy: is 1:1 fresh frozen plasma: packed red blood cells the answer? J Trauma 65: 261-270. 